\title{
Hidden Dangers and Preventive Measures in the Inventory of Articles in Operating Room
}

\author{
Huang Yi, Yajuan Ke, Hongying Zhu, Suwen Lu, Liurong Cheng, Minghui Liu \\ Hainan General Hospital, Haikou, China \\ Email: g2002m@163.com
}

How to cite this paper: Yi, H., Ke, Y.J., Zhu, H.Y., Lu, S.W., Cheng, L.R. and Liu, M.H. (2021) Hidden Dangers and Preventive Measures in the Inventory of Articles in Operating Room. Open Journal of Preventive Medicine, 11, 192-198.

https://doi.org/10.4236/ojpm.2021.115015

Received: April 15, 2021

Accepted: May 21, 2021

Published: May 24, 2021

Copyright $\odot 2021$ by author(s) and Scientific Research Publishing Inc. This work is licensed under the Creative Commons Attribution International License (CC BY 4.0).

http://creativecommons.org/licenses/by/4.0/

\begin{abstract}
Operating Room is an important place for surgical diagnosis and treatment and rescue of patients. With the rapid development of medical technology, high, precision, sharp continuous development, operating room safety management is particularly important. The error of the counting of the surgical articles causes the foreign body left in the patient's body, which will cause irreversible damage to the patient and even cause the medical dispute and touch the legal problem. Operation items inventory system is an important working system in the operating room. Scientific and standardized methods and systems for counting surgical instruments and dressings during operation can prevent foreign bodies from being left in the body, prevent errors and accidents in the operating room, and are very important for ensuring the safety of the operation of patients. In order to ensure the safety of patients, it is necessary to ensure the correct inventory of surgical items. The factors affecting the accuracy of item counting in the operating room and the preventive measures are summarized as follows.
\end{abstract}

\section{Keywords}

Operating Room, Articles, Hidden Danger of Safety, Preventive Measures Reference

\section{Introduction}

Operating room is an important place for surgical treatment and patient rescue. With the rapid development of medical technology, there are more and more difficult operations, and the safety management of operating room is particularly important. With the rapid development of medical technology, more and more difficult operations, operating room safety management is particularly important. As People's legal concept and self-protection consciousness are increasing, the 
forgetting of foreign objects in patients' bodies has been a difficult problem that perplexes the medical and health industry. On the one hand, it causes great physical and mental harm to patients, on the other hand, the surgical team performing the operation will face medical error damages and lawsuits. Because of the particularity of the operation center, the nursing work of the nurse in the operation room is closely related to the medical work of the surgeon and anesthesiologist at the same time [1]. At the same time, the extensive application of new medical technology, the constant investment of new instruments and equipment and the uncertainty of medicine itself also increase the probability of interruption of nursing work in operating room. The counting system of surgical articles is an important working system in operating room. Scientific and standardized methods and systems for counting surgical instruments and dressings during operation can prevent foreign bodies from being left in the body, prevent errors and accidents in the operating room, and are very important for ensuring the safety of the operation of patients. In order to ensure the safety of patients, it is necessary to ensure the correct inventory of surgical items. The factors affecting the accuracy of item counting in the operating room and the preventive measures are summarized as follows.

\section{Causes of Potential Safety Hazards}

1) Lack of safety consciousness, paralysis of thinking knowledge, some doctors and nurses have no correct understanding of safety of goods inventory, and do not face up to the seriousness of goods left behind; or some doctors do not cooperate with the operating room nurse's work arrangement, in the operation process the article carelessly throws about, carelessly takes the operating table the instrument. Some minor surgery such as superficial debridement suture, tumor excision, since that Gauze, stitches, stitches will not leave the body, to the operation security risks [2]. Weak Safety Awareness: medical staff often lack of awareness of the importance of inventory, especially the junior nurses, a strong sense of responsibility, less relevant work experience, lack of predictability of potential risks.

2) Lack of experience, neglect of the importance of inventory, rotation time of new nurses in operating room is too fast, the whole group of nurses is in the state of low seniority. The operation itself is a linked process, each step is related to the smooth operation, the new nurse's working experience is not enough, and there is not enough understanding of many working links, safety awareness, operation cooperation, etc., movement speed often can not keep up with the doctor, often in the busy rhythm easy to ignore or even lost items. At the same time for the experience-related cases are few, easy to ignore the importance of inventory items.

3) The time is tight, the small items can not be packed in time when the counter is opened. During the process of sterilization and inventory by the doctor at the beginning of surgery, often left some wrapped items of rubber bands, silk 
thread small pieces of paper, blade wrapping paper, not in time before the skin clean, and not included in the inventory of items, easy to leave. Operating Room less staff less receiving operation more, large workload for a long time, easy to cause the nurses tired attention distraction. The operating nurse may not change the person midway, when the special situation changes the person, hand over the number and so on clean instrument, dressing, joint signature.

4) The small and sharp instruments do not have matching magnetic box to be placed. Needles, syringes and disassembly blades are often found scattered on the instrument cart and are not contained in a fixed container, easy to be taken away from the operating table in a tense moment, resulting in the loss of stitches.

5) Details of the inspection is not serious, staff is too tired, unpacked after finishing equipment without inspection basin, bowl, small cup habit. Before using the container to load other items, do not check whether there are other items inside, resulting in repeated search during the inventory process, do not know where to go. Sometimes even the operation time is too long, resulting in lack of concentration. Operating Room Labor intensity, even more than the operation, equipment nurses standing for a long time, traveling nurses, physical and mental stress are larger, easy to fatigue Hypoxia, lack of concentration, resulting in error defects.

6) There is a lack of coordination between the doctors and nurses, some of whom do not assist the nurses in the inventory of the items, but rather close the incisions in order to finish the operation quickly, and if problems are found before the items are checked correctly, will not take the initiative to assist the nurse's inventory, and often questioned the nurses, to the safety of the nurses inventory items brought obstacles. The same room receives a table operation, anesthesiologist, the chief surgeon excessively urges, the article clean not cleanly, has the remnant. There are differences in the timing and content of the inventory among different nurses, and the standards are not uniform.

7) The improper design of the sump containers and the location of the containers under the stage for holding the dirt are too far and too low, resulting in the frequent occurrence of small dressings, cotton balls and pieces of cotton wool being thrown outside, coupled with the movement of the staff, easy to cause the loss of items. The needle, syringe needle and disassembly blade are often scattered on the instrument cart, and there is no fixed container to hold them. Especially, the new nurse likes to place the needle and thread, so it is easy to be taken away from the operating table in the tense moment, which causes the loss of the needle.

8) The operating environment is untidy, the indoor floor is too messy and the garbage pail is too small and full after the operation begins. Some nurses and doctors like to throw the packing bag, silk thread and silk thread packing shell randomly, everywhere and at any time. A lot of rubbish can be found on the ground, even around the dustbin are all kinds of rubbish, which brings inconvenience to the inventory of articles after the operation. Removing the contents of 
the room in the middle of surgery. During the same operation, the patient changed position and took the container out of the operating room without checking by the traveling and instrument nurses. Surgical instruments or items left on the floor may be mixed with the next surgical instrument item, which may result in a wrong count, which is more likely to occur when the operation is performed together. Because tidies up the environment the time to be urgent even to have no time tidies up, thus causes next operation item to count confusedly.

9) The counting method of articles is not standardized and perfect, and the inspection of the small parts of the same operation is not serious, such as the integrity of each puncture instrument nut, the plastic sheet of the ultrasonic knife head, each screw of the orthopaedic instrument, etc. After each appliance is used, check first to prevent the accessories from falling off in the body [3]. Kept the nurses busy with writing and neglected to manage the operating table. The contents of the inventory does not give enough attention to small items, there is a fluke psychology.

\section{Preventive Measures}

\subsection{Strengthening Staff Management}

1) Strengthening safety awareness and strengthening work responsibility education for each member of the surgical team, it should be fully recognized that the work of surgical inventory is a necessary responsibility [4] [5]. Managers should carry out safety warning education, work responsibility education and law education repeatedly.

2) Pay attention to the communication and coordination between doctors and nurses to count the instruments and dressings is one of the duties of the surgeons. Communication with doctors should be strengthened in daily work, so that they realize that the inventory is not just a nurse's job, so as to cooperate fully.

3) Rational scheduling, physical and mental stress relief the nature of the work of the operating room determines that nurses are often in a state of fatigue. As head nurse, I take the initiative to care for their physical and mental state and family situation, in case of physical reasons and special circumstances as far as possible to arrange their leave. At the same time, young nurses are reminded not to stay up too late at night to ensure adequate sleep in surgery to be energetic.

\subsection{Standardized Counting Process, System}

1) Standardized counting process surgical items counting system requires hand washing, itinerant nurses should strictly sing in pairs at three key points: before the operation, before closing the body cavity, and after closing the body cavity, I think it's important to do an inventory before surgery. If the count is not in line, you should check it again and report it to the surgeon-in-charge. If necessary, you should report to the head nurse. If the count is still out of line, you can use the c-arm machine to look it up, after fluoroscopy, we still can not find a common analysis with the surgeon to determine that no body cavity left before the 
end of the operation, and in the department error defect register to record the incident, solo surgery, hand washing, tour tripartite signature.

2) Timely recording of additions there was an incident in the Department of laparoscopic surgery in which the surgeon forgot to remove a small piece of Gauze (a temporary addition to the surgical procedure) when the surgeon inserted a small piece of gauze into the abdominal cavity and the scrub nurse failed to alert the ambulatory nurse, and the traveling nurse didn't keep track. Fortunately, the surgeon closed the incision in time to remember, in general anesthesia did not wake up in time to take out the small gauze, otherwise the consequences can not be imagined. Therefore, the temporary addition or placement of items in the cavity during the operation must be recorded in a timely manner, at the same time, medical care to remind each other.

\subsection{Pay Attention to the Factor Control of Surgical Objects}

1) When counting objects, the hand-washing nurse and the tour nurse should have a strict working attitude, strictly enforce the system of two-person singing point, perfect the counting content, the objects on the stage should be counted. The department has applied for the use of developing gauze for fluoroscopy if necessary to find and confirm the presence of residual body cavity.

2) Most of the missing stitches were caused by improper operation. The hand-washing nurse should have a high sense of responsibility, pay attention and know where the stitches went. Remind the surgeon of the procedure and hold the needle in the needle-holding forceps during needle transfer. The deformed stitches are replaced immediately. Break in time to find, if necessary with $\mathrm{C}$-arm machine perspective or film. Many countries such as the United States, Japan and other countries have taken $\mathrm{x}$-rays of surgical patients before going out of the room as a routine item to reduce the risk of foreign body left after surgery.

3) In order to avoid the loss of parts and components of surgical instruments, the hand-washing nurse must wash hands in advance, check the integrity of the instruments together with the visiting nurse, whether the screws and nuts are loose or falling off, and whether the bite of the bone forceps is missing, the surgical field should be kept clean, the instruments should be placed in order, and the integrity should be checked immediately after use.

4) Before the commencement of minimally invasive surgery, make a routine inventory of the items and do a good job of registration. When transferring to Laparotomy, make sure that the items related to minimally invasive surgical instruments are checked and cleared, and then prepare the items for laparotomy or thoracotomy again to avoid confusion.

\subsection{Attach Importance to the Management of Environmental Factors}

1) Standardize the placing of instruments hand-washing nurses as the first important role in the inventory management of instruments and instruments [6] 
[7]. During the operation, hand-washing nurses should be active, accurate and fluent in the delivery of instruments, surgical instruments should be kept in good order at all times. Once with a practice nurse to build a platform, before the operation began, items inventory error, closed before the abdomen found a missing needle, repeated search, finally found this needle under the elbow. The reason is that the nurses are not familiar with the operation procedure and the instruments are placed in confusion. Therefore, it is important for nurses to be familiar with the routine of surgical nursing, to know the general procedure and the key points of cooperation. Recommend the use of magnetic suction needle board, teachers must have a high sense of responsibility, let go.

2) To overcome the shortage of time for counting instruments the nurse should prepare the instrument table 20 - $30 \mathrm{Min}$ in advance for brushing hands in case of emergency and critical patients [8]. Supply equipment items in addition to the name of the correct quantity, be sure to pay attention to accessories are complete, and properly placed.

3) Timely clearance of medical waste to avoid the impact of medical items generated from pre-disposal such as anaesthesia and skin disinfection on the follow-up inventory, now uniform provisions of the department before cutting the skin mobile nurses must be in time to clean up the pre-garbage, so that follow-up inventory is not affected.

\section{Conclusion}

Operating room nurses play an important role in the counting process, and numerous small defects, hidden dangers and loopholes may be gathered together to cause a big mistake accident. Therefore, the work of the dribs and drabs for summary, improvement, eliminate hidden dangers, eliminate errors, accidents. The quality of surgical inventory is directly related to the safety of surgical patients. Therefore, it is necessary to strengthen the safety education and the study of the work responsibility system of the operating room nurses, strengthen the work responsibility, improve the compliance of the counting system, and strengthen the key supervision and strict examination in management, including the details that may cause foreign body to be left in the quality assessment [9] [10] [11]. After the implementation of the comprehensive management measures, the sense of responsibility of the staff and the standardization of the counting system are strengthened, the errors of the counting of the surgical items are eliminated, and the quality of nursing care is improved.

\section{Funding}

2020 Hainan province health industry scientific research project (No. 20A200128).

\section{Conflicts of Interest}

The authors declare no conflicts of interest regarding the publication of this paper. 


\section{References}

[1] Pan, J.R. and Zhang, X.H. (2017) Application of Instrument Checklist in Surgical Safety Management. Nursing Practice and Research, 14, 92-93.

[2] Gu, J.H., Zhang, J.Y. and Xu, H. (2016) Evaluation on the Effect of Centralized Management of Dental Instruments in Disinfection and Supply Center with Traceability System. Chinese Journal of Hospital Infection, 26, 5246-5248.

[3] Wang, H.M., Zhong, L.H., Xiang, P., et al. (2016) A Study on Quality Control Loop to Improve the On-Time Delivery Rate of Foreign Medical Instruments in Orthopaedic Surgery. Chinese Journal of Hospital Infection, 26, 5007-5009.

[4] Lin, S.Q., Gilbert, B. and Liu, M. (2013) Application of Audit Model to Prevent Inadvertent Foreign Bodies. China Nursing Administration, 2013, 57-59.

[5] Gao, Q. and Lu, Y.J. (2014) Analysis of Vulnerability Factors in the Disposal of Ophthalmic Microinstruments by Fishbone Diagram. Journal of Qiqihar Medical University, 35, 621-622.

[6] Lin, P. and Ma, Y.L. (2016) Analysis and Improvement of Wet Pack Phenomenon of External Medical Instruments in Sterilization and Supply Center. Nursing Practice and Research, 13, 131-132.

[7] Liu, B.H., Zhu, D.J. and Guan, W.Y. (2017) Effect of Intensive Quality Control and Continuous Improvement on Improving the Quality of Surgical Instrument Cleaning and Sterilization. Nursing Practice and Research, 14, 98-100.

[8] Xu, S.J. (2014) On the Application of Details in the Inventory of Goods. Chinese Medicine Guide, 2014, 388-389.

[9] Li, R. and He, L. (2013) Current Situation Analysis and Countermeasures of the Inventory Management of Surgical Articles in Operating Room. PLA Journal of Nursing, 30, 54-56.

[10] Jiang, J.L. and Zhou, L.B. (2020) Application of PDA Mobile Technology in Integrated Sharing of Surgical Instrument Quality Tracking System and Instrument Inventory Data. Journal of Chinese Medicine Administration, 38, 53-54.

[11] Chen, Y. (2020) Observation on the Effect of the Improvement of the Cleaning and Disinfection Process of Endoscopic Instruments. Journal of Clinical Rational Drug Use, 13, 164-165. 